\title{
Role of hydrogen bonding in the catalytic reduction of nitric oxide
}

\section{$\operatorname{AUTHOR}(\mathrm{S}):$}

Shiotari, Akitoshi; Hatta, Shinichiro; Okuyama, Hiroshi; Aruga, Tetsuya

\section{CITATION:}

Shiotari, Akitoshi ...[et al]. Role of hydrogen bonding in the catalytic reduction of nitric oxide. Chemical Science 2014, 5(3): $922-926$

\section{ISSUE DATE:}

2014-03

URL:

http://hdl.handle.net/2433/197316

\section{RIGHT:}

This journal is (C) The Royal Society of Chemistry 2014.; This is not the published version. Please cite only the published version.; この論文は 出版社版でありません。引用の際には出版社版をご確認ご利用くださ い。 


\title{
Role of hydrogen bonding in the catalytic reduction of nitric oxide ${ }^{\dagger}$
}

\author{
Akitoshi Shiotari, ${ }^{a}$ Shinichiro Hatta, ${ }^{a}$ Hiroshi Okuyama, ${ }^{a}$ and Tetsuya Aruga ${ }^{a}$
}

\author{
Received Xth XXXXXXXXXX 20XX, Accepted Xth XXXXXXXXX 20XX \\ First published on the web $X$ th $X X X X X X X X X X 200 X$ \\ DOI: 10.1039/b000000x
}

Heterogeneous catalysis is inherently complex, and this makes it difficult to trace the reaction and clarify the mechanism. In this study, we investigated the reduction of nitric oxide (NO) by water on $\mathrm{Cu}(110)$ in a well-defined environment. Scanning tunnelling microscopy was used to control and image the reaction, and to characterize the product and the intermediate. A oneto-one reaction yields a characteristic NO-water complex, in which water induces partial filling of the empty $2 \pi^{*}$ orbital of $\mathrm{NO}$, leading to $\mathrm{N}-\mathrm{O}$ bond weakening. Subsequent reaction of the complex with another water molecule induces further weakening of the $\mathrm{N}-\mathrm{O}$ bond, leading to bond rupture. We reveal that hydrogen-bond coupling induces back-donation and thus plays a crucial role in $\mathrm{N}-\mathrm{O}$ bond cleavage; this provides a fundamental insight into the catalytic reduction of NO under ambient conditions.

\section{Introduction}

The catalytic reduction of nitric oxide (NO) from exhaust streams plays an important role in the removal of air pollution. ${ }^{1,2}$ Although it is challenging to unravel the catalytic processes and understand the mechanism, the elementary processes have been investigated using single-crystalline metal surfaces as model systems to provide atomic-scale insights into the catalysis. ${ }^{3}$ Scanning tunnelling microscopy (STM) has been used to investigate the adsorption and reaction of NO on metal surfaces. ${ }^{4-7}$ STM can identify the 'active site' that is responsible for the catalytic activity by imaging on-going reactions at the surfaces. Zambelli et al. revealed the essential role of atomic steps as active site for the dissociation of NO on $\mathrm{Ru}(0001) .{ }^{4}$ It is feasible to control the reaction in a stepby-step manner, enabling access to intermediate species, using STM as a tool to manipulate individual molecules on metal surfaces. Furthermore, STM can characterize and identify products by single-molecule electronic and vibrational spectroscopies. ${ }^{8}$ In this work, we induced the reaction of NO with water on $\mathrm{Cu}(110)$ by manipulating individual molecules, and used STM spectroscopic techniques to characterize the intermediate as well as the product. A water molecule perturbs the valence state of NO in such a way that the empty $2 \pi^{*}$ state is down-shifted near to the Fermi level, so it is partially filled. As a consequence, the reaction with two water molecules induces $\mathrm{N}-\mathrm{O}$ bond cleavage, yielding a chain of $\mathrm{OH}$ and $\mathrm{NH}$ groups on the surface. Our results show that hydrogen bond-

\footnotetext{
$\dagger$ Electronic Supplementary Information (ESI) available: the formation process of a NO-OH complex and detailed analysis of the $\mathrm{d} I / \mathrm{d} V$ of a $(\mathrm{OH})_{2} \cdots \mathrm{NH}-\mathrm{OH}$ product. See DOI: $10.1039 / \mathrm{b} 000000 \mathrm{x} /$

a Department of Chemistry, Graduate School of Science, Kyoto University, Kyoto 606-8502, Japan. Fax: +81 75753 4000; Tel: +81 75753 3977; E-mail: hokuyama@kuchem.kyoto-u.ac.jp
}

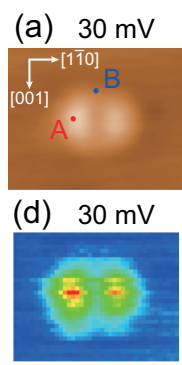

(f) $2 \pi^{*} a$

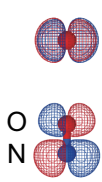

(b) $300 \mathrm{mV}$

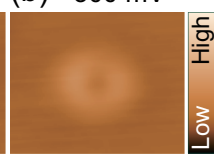

(e) $300 \mathrm{mV}$

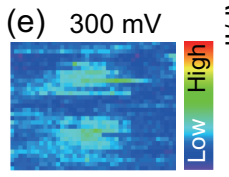

$2 \pi^{*}{ }_{b}$

Top View Side View $[1 \overline{1} 0] \longrightarrow$

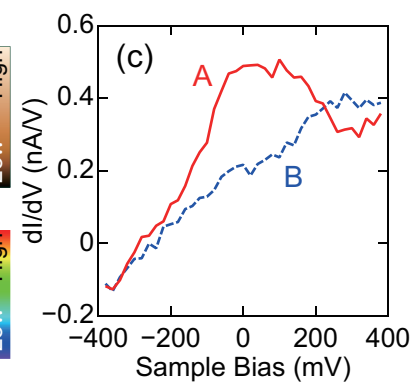

(g)

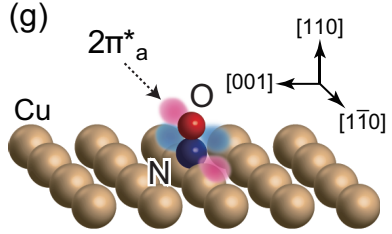

Fig. 1 Topographic STM image of $\mathrm{NO}$ on $\mathrm{Cu}(110)$ at (a) $V=30$ $\mathrm{mV}$ and (b) $V=300 \mathrm{mV}(I=0.5 \mathrm{nA})$. (c) $\mathrm{d} I / \mathrm{d} V$ recorded over position $\mathrm{A}$ in (a) (solid curve), and that recorded over position $\mathrm{B}$ (dashed curve). The spectra were obtained using a tip height corresponding to $V=30 \mathrm{mV}$ and $I=0.05 \mathrm{nA}$ over the protrusion. $\mathrm{d} I / \mathrm{d} V$ spatial maps recorded at (d) $V=30 \mathrm{mV}$ and (e) $V=300 \mathrm{mV}$. The spatial maps were obtained at a constant height corresponding to $V=30 \mathrm{mV}$ and $I=0.5 \mathrm{nA}$ for (d), and $V=30 \mathrm{mV}$ and $I=0.05$ $\mathrm{nA}$ for (e), over the protrusions. (f) Schematic illustrations of the two orthogonal $2 \pi^{*}$ orbitals, namely the $2 \pi_{a}^{*}$ orbital with the lobe along the [1 $1 \overline{1} 0]$ direction (left) and $2 \pi_{b}^{*}$ orbital along the [001] direction (right), viewed from the top and side. (g) Schematic illustration of $\mathrm{NO}$ on $\mathrm{Cu}(110)$ with the $2 \pi_{a}^{*}$ orbital. NO is adsorbed upright at the short-bridge site via an $\mathrm{N}$ atom. The 'dumb-bell' shape in (a) indicates that the $2 \pi_{a}^{*}$ orbital dominates the STM image at $V=30 \mathrm{mV}$, and the 'ring' shape in (b) indicates that $2 \pi_{a}^{*}$ and $2 \pi_{b}^{*}$ orbitals both contribute to the image at $V=300 \mathrm{mV}$. The image sizes are $18 \times 14 \AA^{2}$ for (a) and (b), and $13 \times 10 \AA^{2}$ for (d) and (e). 

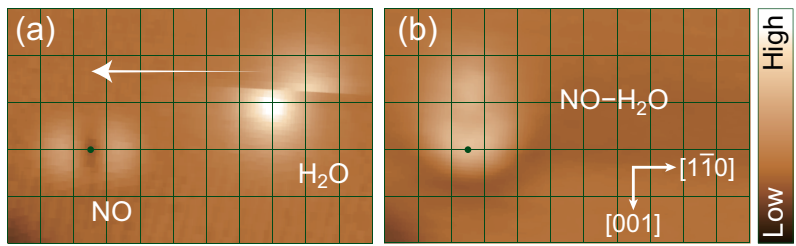

(c)

(d)

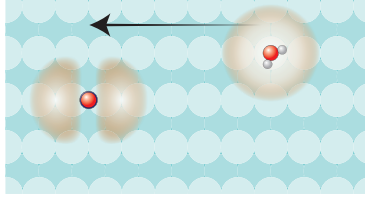

Fig. 2 (a) STM images of NO and water molecules located on neighbouring rows $(V=30 \mathrm{mV}, I=0.5 \mathrm{nA})$. (b) STM image of NO-water complex produced by manipulating the water molecule to NO, as depicted by an arrow in (a). The lines show the lattice of $\mathrm{Cu}(110)$. Schematic illustration of molecules and their appearance for (a) and (b) are shown in (c) and (d), respectively. White, red, and blue spheres represent $\mathrm{H}, \mathrm{O}$, and $\mathrm{N}$ atoms, respectively. The $\mathrm{NO}$ molecule appears as a 'dumb-bell' shape in (a), but it turns into a 'crescent' shape in (b), suggesting a change in the valence state on interaction with a water molecule. The image size is $28 \times 18 \AA^{2}$.

ing with water induces back-donation from the substrate to the antibonding $2 \pi^{*}$ orbital, facilitating $\mathrm{N}-\mathrm{O}$ bond rupture.

\section{Methods}

The STM experiments were carried out in an ultrahighvacuum chamber at $5 \mathrm{~K}$ (USM-1200, Unisoku). An electrochemically etched tungsten tip was used as an STM probe. Single-crystalline $\mathrm{Cu}(110)$ was cleaned by repeated cycles of $\mathrm{Ar}^{+}$sputtering and annealing. The surface was sequentially exposed to $\mathrm{NO}$ and $\mathrm{H}_{2} \mathrm{O}$ gases via a tube doser positioned $\sim 1$ $\mathrm{cm}$ from the surface, at $12 \mathrm{~K}$, through a variable-leak valve.

For characterization of the valence states, scanning tunnelling spectra $(\mathrm{d} I / \mathrm{d} V)$ were recorded over the molecules using a lock-in amplifier with a modulation voltage of $4 \mathrm{mV}_{\text {rms }}$ at $590 \mathrm{~Hz}$, and displayed after subtraction of $\mathrm{d} I / \mathrm{d} V$ recorded over the clean surface at the same tip height. The $\mathrm{d} I / \mathrm{d} V$ spatial maps were obtained at a fixed bias voltage with the tip height kept constant.

The vibration spectra provide information about chemical structure of the adsorbates. The inelastic electron tunnelling spectroscopy with STM has proved to be a powerful tool to identify the adsorbed species at a single-molecule level. ${ }^{9}$ The vibration excitation opens an additional channel of tunnelling electrons and usually gives rise to a peak at the corresponding voltage in the $\mathrm{d}^{2} I / \mathrm{d} V^{2}$. The vibration excitation can also induce dynamical motion of adsorbates, which results in the peak and/or dip in the $\mathrm{d} I / \mathrm{d} V .{ }^{10-13}$ We employed the $\mathrm{d} I / \mathrm{d} V$ to

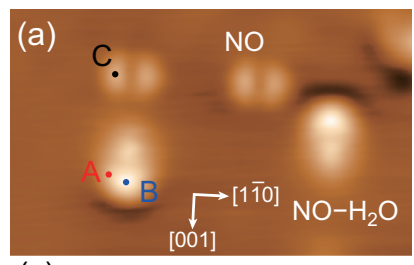

(c)
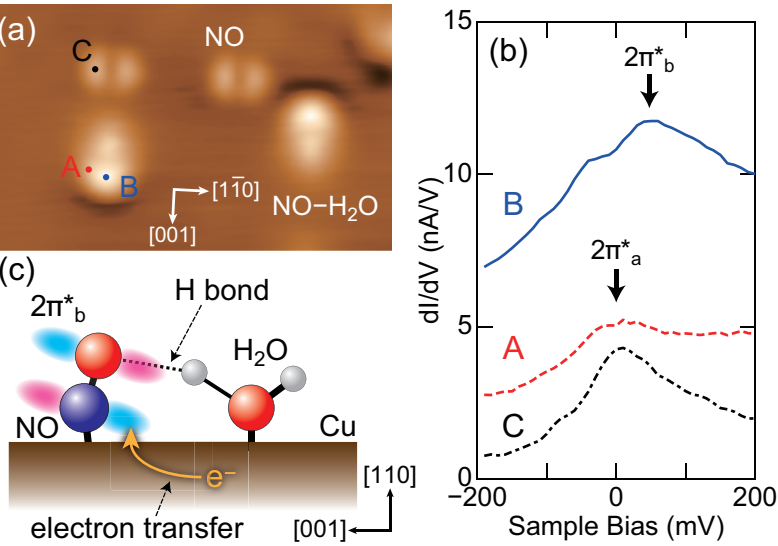

Fig. 3 (a) STM images of isolated NO molecules and NO-water complexes on $\mathrm{Cu}(110)$. (b) $\mathrm{d} I / \mathrm{d} V$ recorded over position $\mathrm{A}-\mathrm{C}$ in (a), with a tip height corresponding to $V=30 \mathrm{mV}$ and $I=0.5 \mathrm{nA}$. (c) Schematic illustration of NO-water complex (side view). $\mathrm{d} I / \mathrm{d} V$ in (b) indicates that the $2 \pi_{b}^{*}$ level is down-shifted and partially filled in the complex, suggesting that water donates a hydrogen bond to NO, which induces electron transfer from the substrate to the $2 \pi_{b}^{*}$ orbital. Thus, NO and water form a hydrogen-bonded complex, as illustrated in (c). The image size in (a) is $48 \times 30 \AA^{2}$.

characterize and identify the reaction product of $\mathrm{NO}$ with water molecules. The modulation voltage of $6-12 \mathrm{mV}_{\mathrm{rms}}$ at 590 $\mathrm{Hz}$ was used for vibrational $\mathrm{d} I / \mathrm{d} V$, and the spectra were displayed without background subtraction.

\section{Results and discussion}

Figure 1a shows a typical topographic image of an isolated NO molecule on $\mathrm{Cu}(110)$, recorded at a sample bias $V=30 \mathrm{mV}$ and tunnelling current $I=0.5 \mathrm{nA}$. The molecule is bonded to the short-bridge site in an upright configuration, ${ }^{14}$ and appears as a dumb-bell-shaped protrusion aligned along the [110] direction ( $\mathrm{Cu}$ row direction). The originally degenerate $2 \pi^{*}$ orbital is split into two levels, namely $2 \pi_{a}^{*}$ and $2 \pi_{b}^{*}$ (Fig. 1f), on the two-fold $\mathrm{Cu}(110)$ surface. The $2 \pi_{a}^{*}$ orbital whose lobes are aligned along the $\mathrm{Cu}$ row direction is imaged by STM at $V=$ $30 \mathrm{mV}$ (Fig. 1a), as shown schematically in Fig. $1 \mathrm{~g}$. The $\mathrm{d} / / \mathrm{d} V$ spectrum taken over the protrusion (A in Fig. 1a) shows a peak at the Fermi level (solid curve in Fig. 1c), indicating that the $2 \pi_{a}^{*}$ level is nearly half-filled. In contrast, the $\mathrm{d} I / \mathrm{d} V$ spectrum taken over the node (B in Fig. 1a) shows a monotonic increase to $400 \mathrm{mV}$ (dashed curve in Fig. 1c). This implies that the $2 \pi_{b}^{*}$ counterpart is located above $400 \mathrm{meV}$ with respect to the Fermi level, and therefore is almost empty. This is confirmed by the $\mathrm{d} I / \mathrm{d} V$ mapping recorded at $V=300 \mathrm{mV}$ (Fig. 1e), which shows the shape of the $2 \pi_{b}^{*}$ orbital, whereas that recorded at $V=30 \mathrm{mV}$ shows the shape of the $2 \pi_{a}^{*}$ orbital (Fig. 1d). The topographic image recorded at $V=300 \mathrm{mV}$ (Fig. 1b) is con- 

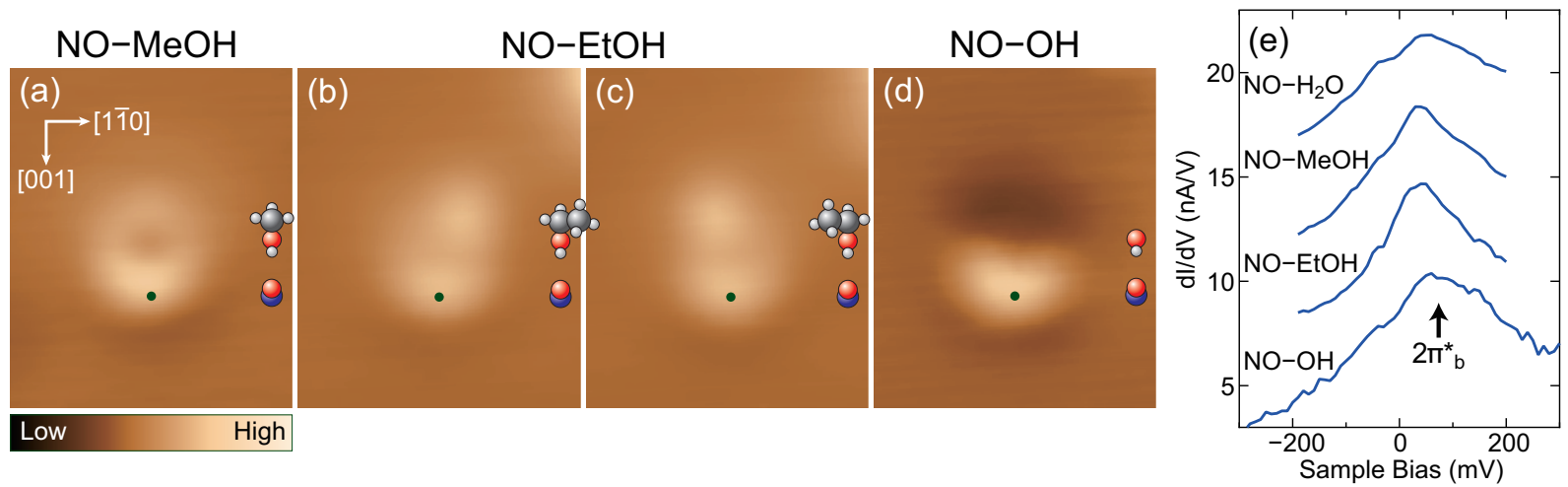

Fig. 4 STM images of (a) NO-methanol, (b),(c) NO-ethanol, and (d) NO-OH complexes ( $V=30 \mathrm{mV}, I=0.5 \mathrm{nA})$, and schematic diagrams of their structures. Two equivalent configurations are observed for the NO-ethanol complex [(b) and (c)], and this is ascribed to tilting of the ethyl group, as shown in the inset. The tilt direction can be changed by a voltage pulse of $150 \mathrm{mV}$ by the STM. In all the complexes, the NO molecule has a 'crescent' shape, as in the NO-water complex (Fig. 2b). (e) dI/dV recorded over the NO molecule (dots) in the complexes. For comparison, $\mathrm{d} I / \mathrm{d} V$ for the NO-water complex is also shown. In all cases, the $2 \pi_{b}^{*}$ level is down-shifted and partially filled, indicating that the complexes are stabilized by hydrogen-bond interactions via partial filling of the $2 \pi_{b}^{*}$ orbital. Each spectrum is vertically offset for clarity. The image sizes are $18 \times 22 \AA^{2}$.

tributed by $2 \pi_{b}^{*}$ as well as $2 \pi_{a}^{*}$, giving rise to the superposed ring shape.

The reaction with a water molecule alters the valence state of a NO molecule via electrostatic hydrogen-bond interactions. Figures $2 \mathrm{a}$ and $\mathrm{b}$, respectively, show topographic images of NO before and after reaction with a water molecule, along with their schematic representations (Figs. 2c and d). An isolated water molecule is adsorbed flat on the top of the $\mathrm{Cu}$ atom and diffuses along the $\mathrm{Cu}$ row, ${ }^{15}$ which causes a fractional image, whereas a NO molecule is bonded to the short-bridge site on the next row (Fig. 2a). By manipulating the water molecule along the $\mathrm{Cu}$ row to $\mathrm{NO},{ }^{16}$ we produce a $\mathrm{NO}$-water complex bonded along the [001] direction (Fig. 2b). The complex has a mirror plane along the [001] direction and therefore has $C_{S}$ symmetry; this suggests that the water molecule is standing up at the short-bridge site (Fig. 2d), as observed in the water-OH (hydroxyl) complex. ${ }^{16}$ The complex can be dissociated by a voltage pulse of $V=0.3 \mathrm{~V}$ by the STM. In the complex, the NO molecule is no longer imaged as a dumb-bell shape but appears as a crescent shape, as illustrated in Fig. 2d, suggesting that the valence state is perturbed by the water molecule.

The $\mathrm{d} I / \mathrm{d} V$ spectra recorded over the NO molecule in the complex (A and B in Fig. 3a) are shown in Fig. 3b, together with that recorded over an isolated NO molecule (C in Fig. 3a). The positions $\mathrm{A}$ and $\mathrm{B}$ correspond to the lobes of the $2 \pi_{a}^{*}$ and $2 \pi_{b}^{*}$ orbitals, respectively, for the original NO molecule. The $\mathrm{d} I / \mathrm{d} V$ recorded over B shows a new peak at $\sim 50 \mathrm{mV}$, indicating that the $2 \pi_{b}^{*}$ state shifts down to $\sim 50 \mathrm{meV}$ as a result of interaction with a water molecule. In contrast, the $\mathrm{d} I / \mathrm{d} V$ recorded over A shows a peak centred at the Fermi level; the $2 \pi_{a}^{*}$ state is not affected by the water molecule. The water molecule is bonded to NO along the [001] direction, and thus the $2 \pi_{b}^{*}$ orbital is selectively perturbed by the water molecule. The topographic image of NO in the complex recorded at $V=$ $30 \mathrm{mV}$ is therefore contributed by the down-shifted $2 \pi_{b}^{*}$ orbital as well as the intact $2 \pi_{a}^{*}$ orbital, resulting in the change of the image from a dumb-bell shape to a crescent one. The structure of the hydrogen-bonded complex is shown schematically in Fig. 3c; NO accepts a hydrogen bond from water and is possibly tilted from the surface normal as a result of the interaction. The electrostatic interaction with water (hydrogen-bond donor) stabilizes the $2 \pi_{b}^{*}$ state near to the Fermi level, leading to partial filling of the orbital (B in Fig. 3b). The NO molecule is therefore negatively charged via electron transfer from the substrate, stabilized by hydrogen-bond interactions with a water molecule. The NO-water interaction is quite weak in the gas phase,${ }^{17}$ highlighting the essential role of electron transfer from the substrate in molecular interactions on metal surfaces. ${ }^{18,19}$

To confirm that hydrogen-bond interactions are responsible for the change in the valence state, we examined the interactions of $\mathrm{NO}$ with methanol, ethanol, and $\mathrm{OH}$ groups on the surface (Fig. 4). An isolated methanol molecule diffuses across the surface, even at $6 \mathrm{~K},{ }^{20}$ and it forms a stable complex with NO, as shown in Fig. 4a. The NO-methanol complex shows similar features to those of the NO-water complex; it has $C_{S}$ symmetry, with the NO molecule imaged as a crescent shape, and the corresponding $\mathrm{d} I / \mathrm{d} V$ shows a peak at $\sim 50 \mathrm{mV}$, at nearly the same voltage as the NO-water complex (Fig. 4e). The down-shift of the $2 \pi_{b}^{*}$ level is therefore caused by hydrogenbond interactions between NO and methanol. We propose that the structure of the NO-methanol complex is as shown in the 


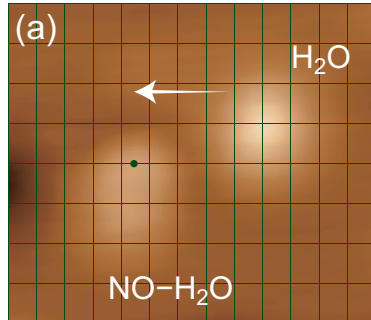

(c)

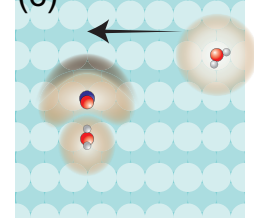

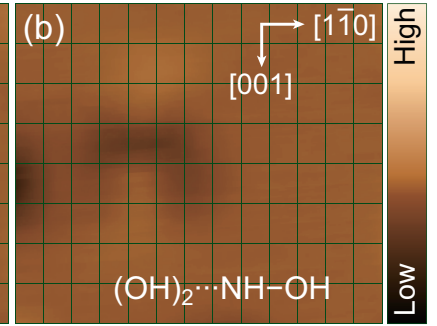

(d)

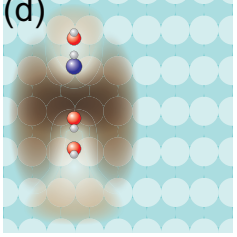

Fig. 5 (a) STM images of a NO-water complex and a water molecule. NO in the complex and the water molecule are located on the neighbouring $\mathrm{Cu}$ rows. (b) STM image of the product yielded by manipulating the water molecule to the complex, as shown by an arrow in (a). Schematic diagrams for (a) and (b) are shown in (c) and (d), respectively. The image sizes are $33 \times 29 \AA^{2}$ for (a) and (b).

inset of Fig. 4a, in which the methanol donates a hydrogen bond to NO along the [001] direction, in a similar way as in the NO-water complex. The NO-ethanol complex does not have $C_{s}$ symmetry, but shows two equivalent configurations (Figs. 4b and c), suggesting that the ethyl group is tilted in the [1 $1 \overline{0}$ ] direction, as shown in the inset. Nevertheless, the common feature in the $\mathrm{d} I / \mathrm{d} V$ (Fig. $4 \mathrm{e}$ ) confirms that hydrogen bonding also has a role. A NO-OH complex (Fig. 4d) was produced by manipulating a NO molecule to an immobile $\mathrm{OH}$ group (Fig. S1 in ESI $\dagger$ ). The $2 \pi_{b}^{*}$ peak is positioned at a slightly higher voltage (Fig. 4e), suggesting less hydrogenbond coupling in the $\mathrm{NO}-\mathrm{OH}$ complex. Compared with a water molecule, an $\mathrm{OH}$ group is a poor hydrogen-bond donor on a surface ${ }^{21}$ and this is possibly the origin of the difference in the positions of the $2 \pi_{b}^{*}$ states between the complexes.

Electron transfer from the substrate to NO in the complexes induces weakening of the $\mathrm{N}-\mathrm{O}$ bond as a result of partial filling of the antibonding $2 \pi_{b}^{*}$ orbital. The hydrogen-bond interactions with water can therefore facilitate dissociation of NO on the surface. We investigated the effect of a second water molecule on the NO-water complex. Figures 5a and b, respectively, show topographic images before and after the reaction between a NO-water complex and a water molecule, along with schematic diagrams in Figs. $5 \mathrm{c}$ and d, respectively. The second water molecule can interact with the complex from the opposite side to that of the first water molecule, as shown in Fig. 5a. The centre of the new complex (around the NO molecule) is drastically changed from the protrusion to the depression on the reaction with the second water molecule (Fig. 5b). The depression suggests that $2 \pi^{*}$ states of NO are

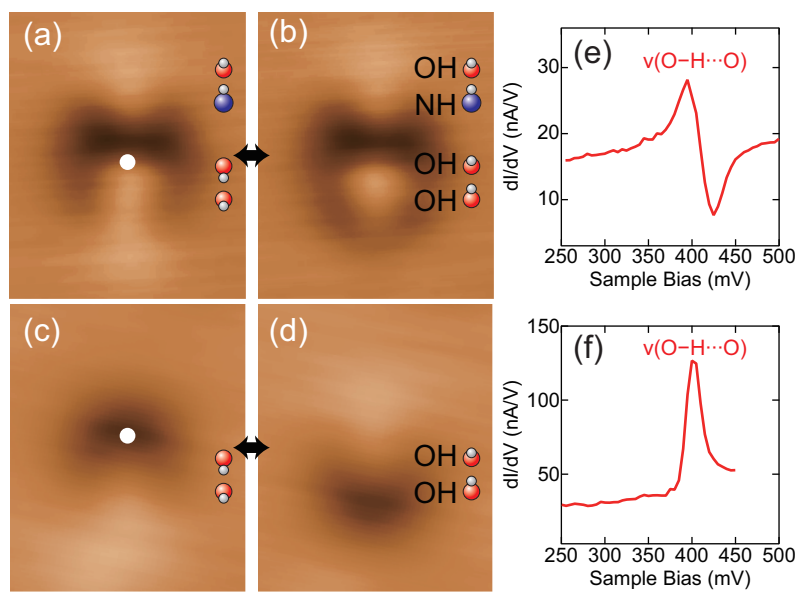

Fig. 6 (a),(b) The STM images of the product yielded by the reaction of the NO-water complex with another water molecule (Fig. 5b). The product flips between (a) and (b) under bias voltage higher than $\sim 250 \mathrm{mV}$. (c),(d) For comparison, the STM images of $(\mathrm{OH})_{2}$ on $\mathrm{Cu}(110)$ are shown. The $(\mathrm{OH})_{2}$ also flips between (c) and (d) under the same condition as in (a) and (b). The similarity in the images suggests that the product is composed of $(\mathrm{OH})_{2}$ and $\mathrm{OH}-\mathrm{NH}$, as shown schematically in (a) and (b). (e) The $\mathrm{d} I / \mathrm{d} V$ spectrum of the product recorded with the tip fixed over the dot in (a) at the height corresponding to $V=30 \mathrm{mV}$ and $I=0.5 \mathrm{nA}$ with the modulation voltage of $12 \mathrm{mV}_{\text {rms }}$. (f) The $\mathrm{d} I / \mathrm{d} V$ spectrum of $(\mathrm{OH})_{2}$ recorded with the tip fixed over the dot in (c) at the height corresponding to $V$ $=30 \mathrm{mV}$ and $I=0.5 \mathrm{nA}$ and with the modulation voltage of 6 $\mathrm{mV}_{\text {rms. }}$. The H-bonded $\mathrm{O}-\mathrm{H}$ stretching modes of $(\mathrm{OH})_{2}$ are observed at $\sim 400 \mathrm{mV}$ in both spectra, providing unambiguous evidence for the $\mathrm{N}-\mathrm{O}$ bond dissociation and $(\mathrm{OH})_{2}$ formation. The image sizes are $18 \times 22 \AA^{2}$.

no longer present near the Fermi level in the product; this is ascribed to dissociation of NO, as evidenced below.

It was found that the product shows two-state fluctuation under bias voltage higher than $\sim 250 \mathrm{mV}$ (Figs. 6a and b). This feature is similar to the flip motion of $(\mathrm{OH})_{2}$ induced by the same voltage (Figs. $6 \mathrm{c}$ and d). The $(\mathrm{OH})_{2}$ was produced by the controlled reaction of oxygen atom with a water molecule. ${ }^{13}$ Therefore we suggest that the $\mathrm{N}-\mathrm{O}$ bond is dissociated and $(\mathrm{OH})_{2}$ is formed in the product. This is supported by the $\mathrm{d} I / \mathrm{d} V$ spectrum. The $\mathrm{d} I / \mathrm{d} V$ recorded over $(\mathrm{OH})_{2}$ on $\mathrm{Cu}(110)$ shows a peak at $\sim 400 \mathrm{mV}$ (Fig. 6f), and this was ascribed to the $\mathrm{H}$-bonded $\mathrm{O}-\mathrm{H}$ stretching mode $[v(\mathrm{O}-\mathrm{H} \cdots \mathrm{O})] .{ }^{13}$ Tunnelling electrons from the STM tip excite the $v(\mathrm{O}-\mathrm{H} \cdots \mathrm{O})$ and induce a flip motion of $(\mathrm{OH})_{2}$ (Figs. $6 \mathrm{c}$ and $\mathrm{d}$ ), giving rise to the peak in the $\mathrm{d} I / \mathrm{d} V$. The $\mathrm{d} I / \mathrm{d} V$ recorded over the product also shows a peak at $\sim 400 \mathrm{mV}$ (Fig. 6e); $(\mathrm{OH})_{2}$ is formed in the product. The characteristic peak-and-dip structure arises from the contributions of two different $\mathrm{O}-\mathrm{H}$ stretching modes, i.e., $v(\mathrm{O}-$ $\mathrm{H} \cdots \mathrm{O})$, associated with the two inequivalent configurations in Figs. $6 a$ and $b$, to the spectrum. The peak-and-dip structure in 
the $\mathrm{d} I / \mathrm{d} V$ is analyzed by a recently proposed analytical modeling, ${ }^{22,23}$ which yields vibrational energies of 403 and 428 $\mathrm{meV}$, assigned to $v(\mathrm{O}-\mathrm{H} \cdots \mathrm{O})$, for the orientations in Figs. $6 \mathrm{a}$ and $b$, respectively (Fig. S $†$ ). It is therefore unambiguously shown that the $\mathrm{N}-\mathrm{O}$ bond is cleaved, yielding $(\mathrm{OH})_{2}$ in the product. We propose that the structure of the product is as depicted in the insets of Figs. $6 a$ and $b$. The configuration of the product is represented by $(\mathrm{OH})_{2} \cdots \mathrm{NH}-\mathrm{OH}$, and we infer from the images that the $(\mathrm{OH})_{2}$ flips back and forth by vibrational excitation, but the $\mathrm{NH}-\mathrm{OH}$ groups have a fixed orientation.

It is remarkable that two water molecules can dissociate NO on a surface, even at $12 \mathrm{~K}$. We note that although the reaction is induced by STM, to trace individual processes, exposure of the surface to water and NO at $12 \mathrm{~K}$ leads to spontaneous formation of the dissociation product (Fig. 5b) as well as the NO-water complex (Fig. 2b), indicating that the reaction is almost barrierless. The electron transfer from the metal to NO is induced by intermolecular hydrogen-bond coupling, which is the key mechanism for facile bond dissociation. The bond weakening is governed by the extent of back-donation to the antibonding $2 \pi^{*}$ orbital. The adsorption itself can induce $2 \pi^{*}$ rehybridization, weakening the bond, ${ }^{24}$ but back-donation is enhanced by intermolecular hydrogen-bond coupling with water.

\section{Conclusions}

By precisely controlling individual molecules, we visualized the sequential reaction processes of NO with two water molecules on a metal surface; the product as well as the intermediate were characterized at the single-molecule level. Hydrogen-bond interactions with water cause a change in the valence state of NO, facilitating dissociation. As a consequence, two water molecules can reduce NO almost barrierlessly.

\section{Acknowledgements}

A.S. acknowledges the support of the Japan Society for the Promotion of Science. This research was supported in part by a Grant-in-Aid for Young Scientists (A) (NO. 22685002) from Japan Society for the Promotion of Science.

\section{References}

1 M. Iwamoto and H. Hamada, Catal. Today, 1991, 10, 57.

2 W. S. Epling, L. E. Campbell, A. Yezerets, N. W. Currier and J. E. Parks II, Catal. Rev., 2004, 46, 163.

3 Y. Hu, K. Griffiths and P. R. Norton, Surf. Sci., 2009, 603, 1740.

4 T. Zambelli, J. Wintterlin, J. Trost and G. Ertl, Science, 1996, 273, 1688.

5 K. B. Rider, K. S. Hwang, M. Salmeron and G. A. Somorjai, Phys. Rev. Lett., 2001, 86, 4330.

6 C. T. Harbschleb, S. C. Bobaru and J. W. M. Frenken, Catal. Today, 2010, 154, 61.

7 S. C. Li, P. Jacobson, S. L. Zhao, X. Q. Gong and U. Diebold, J. Phys. Chem. C, 2012, 116, 1887.

8 W. Ho, J. Chem. Phys., 2002, 117, 11033

9 B. C. Stipe, M. A. Rezaei and W. Ho, Science, 1998, 280, 1732.

10 J. Gaudioso, L. J. Lauhon and W. Ho, Phys. Rev. Lett., 2000, 85, 1918.

11 J. A. Gupta, C. P. Lutz, A. J. Heinrich and D. M. Eigler, Phys. Rev. B, 2005, 71, 115416.

12 W. H. A. Thijssen, D. Djukic, A. F. Otte, R. H. Bremmer and J. M. van Ruitenbeek, Phys. Rev. Lett., 2006, 97, 226806.

13 T. Kumagai, M. Kaizu, H. Okuyama, S. Hatta, T. Aruga, I. Hamada and Y. Morikawa, Phys. Rev. B, 2009, 79, 035423.

14 A. Shiotari, Y. Kitaguchi, H. Okuyama, S. Hatta and T. Aruga, Phys. Rev. Lett., 2011, 106, 156104.

15 T. Kumagai, M. Kaizu, H. Okuyama, S. Hatta, A. Aruga, I. Hamada and Y. Morikawa, e-J. Surf. Sci. Nanotechnol., 2008, 6, 296.

16 T. Kumagai, M. Kaizu, H. Okuyama, S. Hatta, A. Aruga, I. Hamada and Y. Morikawa, Phys. Rev. B, 2010, 81, 045402.

17 H. Cybulski, P. S. Żuchowski, B. Fernández and J. Sadlej, J. Chem. Phys., 2009, 130, 104303.

18 S. Gao, J. R. Hahn and W. Ho, J. Chem. Phys., 2003, 119, 6232.

19 J. R. Hahn and W. Ho, J. Phys. Chem. B, 2005, 109, 20350.

20 Y. Kitaguchi, A. Shiotari, H. Okuyama, S. Hatta and T. Aruga, J. Chem. Phys., 2011, 134, 174703.

21 I. Hamada, T. Kumagai, A. Shiotari, H. Okuyama, S. Hatta and T. Aruga, Phys. Rev. B, 2012, 86, 075432.

22 Y. Ootsuka, T. Frederiksen, H. Ueba and M. Paulsson, Phys. Rev. B, 2011, 84, 193403.

23 H. Okuyama, A. Shiotari, T. Kumagai, S. Hatta, T. Aruga, Y. Ootsuka, M. Paulsson and H. Ueba, Phys. Rev. B, 2012, 85, 205424.

24 W. A. Brown and D. A. King, J. Phys. Chem. B, 2000, 104, 2578. 